\author{
F. Heredia $\cdot$ R. Cos $\cdot$ S. Moros $\cdot$ L. Torrabadella $\cdot$ \\ E. Cayuela
}

\title{
Radiological control of Essure placements
}

Published online: 24 July 2004

(C) Springer-Verlag Berlin / Heidelberg 2004

\begin{abstract}
Essure is a microinsert placed bilaterally into fallopian tube lumens under hysteroscopic visualization in patients seeking permanent contraception. Our multicenter clinical trial begun in 1998 required hysterosalpingography (HSG) be performed 3 months after placement to confirm tubal occlusion. The patency rate in satisfactorily placed devices was $4 \%$. All of these underwent a repeat HSG 3-4 months later (due to a patency finding at the previous test). We present the results of our clinical use in patients out of the clinical trial. Of 78 insertions 65 (83.3\%) were considered satisfactory, and the patients underwent pelvic radiography after 3 months. Only one patient had an abnormal radiograph, and an HSG was performed confirming bilateral tubal occlusion. In 12 patients $(15.4 \%)$ the placement was considered unsatisfactory and underwent HSG. Ten confirmed bilateral tubal occlusion, and two had tubal patency. Therefore we repeated HSG 3 months later. We verified tubal occlusion in one patient and unilateral tubal patency in the other. In one patient with a satisfactory placement we performed an HSG that corroborated tubal occlusion. No pregnancies have been reported so far. Therefore we consider tubal occlusion successful in all the satisfactory microinsert placements (83.3\%) after 3 months, performing only pelvic radiography. Additional HSG is required in the remaining $17 \%$ due to an unsatisfactory placement. Of these, $83 \%$ will be occluded after 3 months, increasing the rate to $91.6 \%$ after 6 months. These findings show that it is not necessary to conduct HSG when the bilateral placement is satisfactory and can be replaced by pelvic radiography at 3 months to confirm microinsert retention and location. HSG, with its unnecessary risks, costs, inconvenience and discomfort, can thus be avoided in many patients.
\end{abstract}

F. Heredia $(\bullet) \cdot$ R. Cos $\cdot$ L. Torrabadella

Institut Universitari Parc Taulí,

Sabadell, Spain

e-mail: fheredia@cspt.es

S. Moros - E. Cayuela

Hospital General de Vic,

Vic, Spain
Keywords Essure $\cdot$ Hysterosalpingography $\cdot$ Sterilization

\section{Introduction}

The Essure system (Conceptus, San Carlos, Calif., USA) has revolutionized permanent contraception for women. Under hysteroscopic view, placing Essure is easy and quick, does not require general anesthesia, and can be carried out in an outpatient setting (Fig. 1). It involves the placement of a microinsert in each fallopian tube. The presence of the microinsert induces a local fibrous tissue ingrowth from the surrounding tubal walls, achieving tubal occlusion in 3 months [1]. This system does not produce abdominal incisions or scars, contains no hormones, and permits rapid return to normal activities with a high rate of patient tolerance and satisfaction.

The phase II [2] and pivotal [3] multicenter trials that began in 1998 required patients to use alternative contraception for 3 months after the procedure and then undergo hystersalpingography (HSG) to confirm the proper location of the microinserts and tubal occlusion. They were subsequently able to rely on the Essure method for permanent birth control and cease their alternative contraception method [2]. To date no pregnancies have been reported in clinical trial participants.

In 2001 Conceptus questioned whether HSG was always necessary. HSG involves risks, costs, inconvenience, discomfort, noncompliance, and potential recanalization from the procedure itself. Conceptus under-

Diarneter: 0,8 mm - 2,0 mm

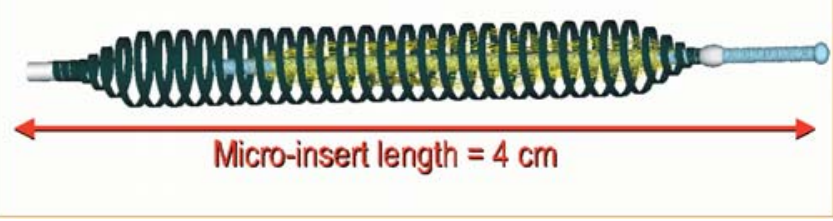

Fig. 1 Microinsert scheme 
Table 1 Findings at follow-up by HSG in phase II [2] and pivotal [3] trials; data as of December 2001

\begin{tabular}{lll}
\hline & Phase II $(n=200)$ & Pivotal $(n=456)$ \\
\hline Satisfactory microinsert location at 3 months & $194 / 200(97 \%)$ & $437 / 456(96 \%)^{\mathrm{a}}$ \\
Bilateral occlusion at 3 months (in patients with satisfactory microinsert location) & $187 / 194(96.4 \%)$ & $421 / 437(96 \%)$ \\
Bilateral occlusion at 6-7 months in patients with satisfactory microinsert location & $194 / 194(100 \%)$ & $437 / 437(100 \%)$ \\
Satisfactory placement and occlusion after replacement of expelled microinsert & n.a. & $9 / 9(100 \%)$ \\
\hline
\end{tabular}

${ }^{a}$ Microinsert expulsions were noted on the HSG in 14 of these 456 women. Nine of these chose to undergo a second procedure, and all 9 had a successful bilateral placement. These nine women are not reflected here as a successful placements, however, since all had unsatisfactory device location at the initial HSG

took a review of the clinical data to date from both the phase II trial of over 200 patients and the pivotal trial of over 500 patients, which included a review of HSGs, radiographs, and videos of all the hysteroscopic device placements in which the HSG detected a potential problem. Based on this review, it was believed that there were clear predictors of potential issues that should be investigated further by HSG. In the absence of these predictors it did not appear necessary to perform HSG routinely. Therefore straightforward criteria were developed for identifying the small proportion of patients who might benefit from an HSG evaluation, based on the evaluation of their 3-month pelvic radiograph (Table 1).

The only exception to this algorithm pertains to detecting tubal patency. The 3-month patency rate in the phase II and pivotal trials was $4 \%$ and $3 \%$ (in study participants with satisfactorily placed devices). All of study participants who underwent repeat HSG at 6-7 months (due to a patency finding at 3 months) were occluded by the second HSG. The 3-month patency rate of $3-4 \%$ is within the range reported in studies of tubal ligation patients. Grunert [4] reported a patency rate of $16.7 \%$ in a study of 54 tubal ligation patients followed up for an average of 4.5 years, and cited literature references for a total of 1,094 patients followed up for 3 months in whom the average patency rate was $3.2 \%$. It should be noted that the pregnancy rates in these studies did not equal the patency rates noted. Therefore it has been reported that despite the possible failure of absolute physical occlusion of the tubes, this does not equate to failure of sterilization $[4,5,6,7]$.

Finally, it is also of note that an HSG is not required in patients who undergo tubal sterilization by any other method, and we believe that the clinical data on the Essure method do not warrant a different requirement in this regard. The 3-month alternative contraception period is still in effect, however, and all patients are required to undergo pelvic radiography at 3 months to confirm device retention and location.

The objective of this study was to determine whether pelvic radiography 3 months after the procedure is sufficient to ensure placement location of the Essure microinsertsin and confirm permanent tubal occlusion.

\section{Design and methods}

Essure is a dynamically expanding microinsert that is placed bilaterally into the proximal fallopian tube lumen in patients seeking

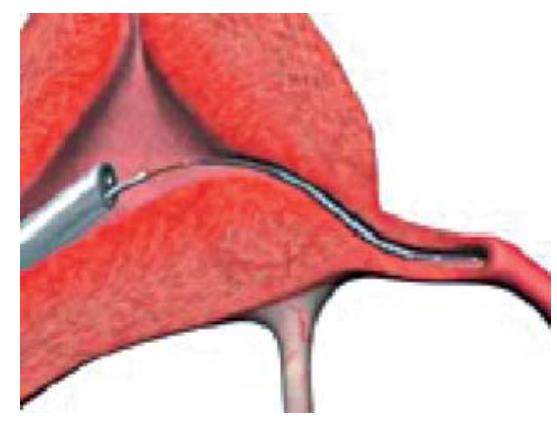

Fig. 2 Microinsert tubal introduction

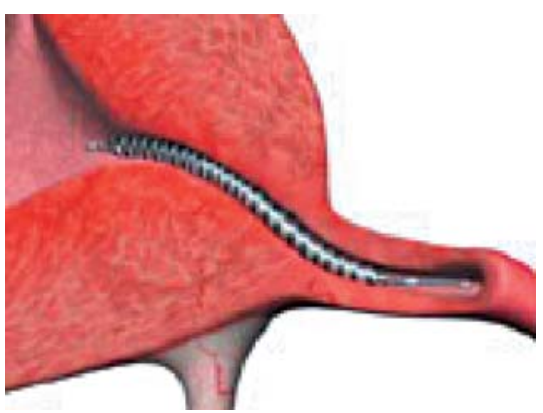

Fig. 3 Microinsert deployment

permanent contraception (Fig. 2). This placement is carried out under hysteroscopic visualization as an outpatient procedure (Fig. 3). We use a rigid hysteroscope, 5-F (1.7 mm internal diameter) operating channel with an outer diameter of $5.5 \mathrm{~mm}$. The microinsert consists of a flexible stainless steel inner coil, a dynamic outer coil made of nickel titanium alloy, and a layer of polyethylene terephthalate fibers running along and through the inner coil [2].

During phase II and phase III multicenter clinical trial satisfactory bilateral insertions was ultimately achieved in 664 of 734 patients $(90 \%)$. Satisfactory placement involved good vision of the tubal ostia and microinsert location across the uterotubal junction, with three to ten visible expanded coils trailing into the uterus. The protocol required that HSG be performed 3 months after placement to confirm tubal occlusion $[2,3]$. The $100 \%$ bilateral tubal occlusion rate indicated that it is not necessary to perform HSG when the initial bilateral placement location is considered satisfactory. Therefore the HSG at 3 months was replaced by pelvic radiography to confirm microinsert retention and location and confirm bilateral tubal occlusion (Figs. 4, 5, 6) [2]. We present the results of our clinical use in patients outside of the scope of the phase II and pivotal clinical trials. We started the clinical use of the Essure microinserts, after enrollment in the clinical trials was completed, in October 2001. To date we have performed 100 procedures. The 


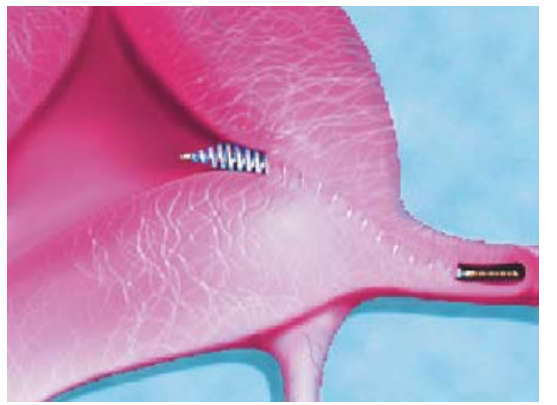

Fig. 4 Three months postplacement under hysteroscopic view

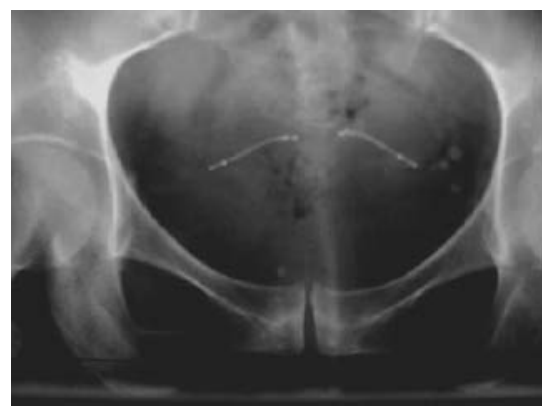

Fig. 5 Microinserts under radiographic view

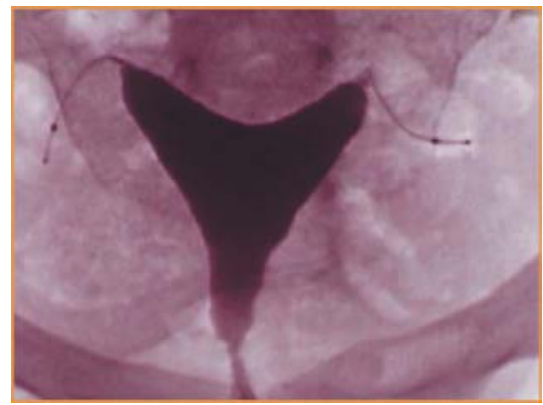

Fig. 6 Hysterosalpingography at 3 months

last 78 have been included in the radiographic protocol. All the procedures were performed with paracervical block only, with $10 \mathrm{ml}$ lignocaine $1 \%$ or, in some cases, with intravenous sedation.

\section{Results}

Sixty-five of the 78 insertions (83.3\%) were considered satisfactory, and patients underwent pelvic radiography 3 months later. Only one patient had a seemingly abnormal radiograph (too much distance between microinserts), and HSG was therefore performed, which confirmed a bilateral tubal occlusion. This represents a $100 \%$ rate of bilateral occlusion when the placement was considered successful. In 12 of the 78 patients $(15.4 \%)$ the placement was considered suboptimal, and all of these underwent HSG. Ten of these patients showed bilateral tubal oc- clusion on HSG, and the remaining two had tubal patency. Therefore we repeated the HSG 3 months later. At this stage we verified complete tubal occlusion in one patient and unilateral tubal patency in the other. The latter underwent a surgical sterilization by laparoscopy. Additionally, in one special patient with only one tube, we achieved satisfactory placement but decided to perform HSG to corroborate tubal occlusion. No pregnancies have been reported to date.

\section{Conclusion}

In our population of 78 patients $83.3 \%$ demonstrated satisfactory device placement at the 3-month postprocedure radiography, and based on the correct position of the microinserts tubal occlusion was assumed. The remaining $17 \%$ had what appeared to be suboptimal placement, and these patients were evaluated further by HSG, which served as a verification tool. In our experience $83 \%$ of these remaining patients demonstrated bilateral tubal occlusion upon HSG 3 months after placement. This rate increased to $91.6 \%$ when the HSG was repeated at 6 months. These figures are in line with clinical trial conclusions $[2,3]$. We conclude that HSG is required only in unsatisfactory placements or special cases. The next step will be to replace the 3-month pelvic radiography with an earlier pelvic ultrasound to demonstrate the correct location of the microinserts to alleviate patient anxiety or diagnose malposition early as other authors propose [8]. Routine HSG can thus be avoided in most patients, along with its unnecessary risks, costs, inconvenience, and discomfort.

\section{References}

1. Valle RF, Carignan CS, Wright TC, STOP Prehysterectomy Investigation Group (2001) Tissue response to the STOP microcoil transcervical permanent contraceptive device: results from a prehysterectomy study. Fertil Steril 76:974-980

2. Kerin JF, Cooper JM, Price T, Van Herendael BJ, Cayuela-Font E, Cher D, Carignan CS (2003) Hysteroscopic sterilization using a micro-insert device: results of a multicentre phase II study. Hum Reprod 18:1223-1230

3. Cooper JM, Carignan CS, Cher D, Kerin JF, Selective Tubal Occlussion Procedure 2000 Investigators Group (2003) Microinsert non incisional hysteroscopic sterilization. Obstet Gynecol 102:59-67

4. Grunert GM (1981) Late tubal patency following tubal ligation. Fertil Steril 35:406-408

5. Cook CL (1982) Evaluation of falope ring sterilization by hysterosalpingogram. J Reprod Med 27:243-245

6. Sheikh HH (1976) Hysterosalpingographic follow-up of laparoscopic sterilization. Am J Obstet Gynecol 126:181

7. Sheikh HH (1977) Hysterosalpingographic follow-up of the partial salpingectomy type of sterilization. Am J Obstet Gynecol 128:858

8. Teoh M, Meagher S, Kovacs G (2003) Ultrasound detection of the Essure permanent birth control device; a case series. Aust N Z J Obstet Gynaecol 43:378 